\title{
可见光促进合成三氟甲基取代的四氢呋喃和四氢吡喃
}

\author{
王 娜 ${ }^{a, b}$ 顾强帅*,c 程永峰 ${ }^{a}$ 李 磊 $a$ \\ 李忠良 ${ }^{c}$ 郭 臻*,b 刘心元 ${ }^{*}, a$ \\ $\left({ }^{a}\right.$ 南方科技大学化学系和格拉布斯研究院 深圳 518055) \\ $\left({ }^{b}\right.$ 太原理工大学材料科学与工程学院 新材料界面科学与工程教育部重点实验室 太原 030024) \\ ( ${ }^{c}$ 南方科技大学前沿与交叉科学研究院 深圳 518055)
}

\begin{abstract}
摘要 利用相对廉价易得、操控简便的三氟甲基磺酰氯为三氟甲基自由基前体，在可见光催化下通过非活化烯烃的自 由基 1,2-烷氧基-三氟甲基化反应实现了一系列三氟甲基化的四氢呋喃和四氢吡喃化合物的高效合成.

关键词 三氟甲基磺酰氯；四氢呋喃；四氢吡喃；可见光；烷氧一三氟甲基化
\end{abstract}

\section{Visible-Light Promoted Preparation of Trifluoromethylated Tetrahydrofuran and Tetrahydropyran}

\author{
Wang, $\mathrm{Na}^{a, b} \quad \mathrm{Gu}$, Qiang-Shuai ${ }^{*, c} \quad$ Cheng, Yong-Feng ${ }^{a} \quad$ Li, Lei ${ }^{a}$ \\ Li, Zhong-Liang ${ }^{c} \quad$ Guo, Zhen ${ }^{*, b}$ Liu, Xin-Yuan*,a \\ ( ${ }^{a}$ Department of Chemistry and Shenzhen Grubbs Institute, Southern University of Science and Technology, Shenzhen 518055) \\ $\left({ }^{b}\right.$ Key Laboratory of Interface Science and Engineering in Advanced Materials, Ministry of Education, College of Materials \\ Science \& Engineering, Taiyuan University of Technology, Shanxi 030024) \\ ( ${ }^{c}$ SUSTech Academy for Advanced Interdisciplinary Studies, Southern University of Science and Technology, Shenzhen \\ 518055)
}

\begin{abstract}
An efficient protocol for facile access to trifluoromethylated tetrahydrofuran and tetrahydropyran has been developed under visible light irradiation conditions via radical 1,2-alkoxyl-trifluoromethylation of unactivated alkene. It features the use of readily commercially available and operatively simple trifluoromethanesulfonyl chloride as a trifluoromethyl radical source, thus making the protocol potentially appealing for practical preparation.

Keywords trifluoromethanesulfonyl chloride; tetrahydrofuran; tetrahydropyran; visible light; alkoxyl-trifluoromethylation
\end{abstract}

\section{Introduction}

Tetrahydrofuran and tetrahydropyran are essential motifs of many natural products and bioactive molecules. ${ }^{[1]}$ Thus, their synthesis has attracted many research efforts over the last several decades. ${ }^{[2]}$ On the other hand, the trifluoromethyl group has recently emerged as a greatly useful tool in pharmaceutical ${ }^{[3]}$ and agrochemical ${ }^{[4]}$ sciences due to its unique beneficial effects for enhancing chemical and metabolic stability, bioavailability, and interaction with bio- logically relevant targets. ${ }^{[5]}$ Therefore, it is highly desirable to develop efficient methods for introducing trifluoromethyl groups ${ }^{[6]}$ into tetrahydrofurans and tetrahydropyrans for potential drug and agrochemical discovery. ${ }^{[7]}$ In this respect, intramolecular 1,2-alkoxyl-trifluoromethylation of unactivated alkene ${ }^{[8]}$ represents a particularly convenient way for accessing various sorts of trifluoromethylated tetrahydrofurans and tetrahydropyrans using different sources of trifluoromethyl radicals, such as Togni's reagents, ${ }^{[9]}$ Umemoto reagents, ${ }^{[10]}$ and trifluoromethyl halides (Scheme

\footnotetext{
* Corresponding authors. E-mail: guqs@sustc.edu.cn; guozhen@tyut.edu.cn; liuxy3@sustc.edu.cn Received August 31, 2018; revised September 26, 2018; published online October 19, 2018. Dedicated to Professor Qingyun Chen on the occasion of his 90th birthday.

Project supported by the National Natural Science Foundation of China (Nos. 21722203, 21831002, 21801116, and 21572096), the Shenzhen Special Funds for the Development of Biomedicine, Internet, New Energy, and New Material Industries (Nos. JCYJ20170412152435366, JCYJ20170307105638498), the Natural Science Foundation of Guangdong Province (No. 2018A030310083) and the Shenzhen Nobel Prize Scientists Laboratory Project (No. C17213101). 国家自然科学基金(Nos. 21722203，21831002，21801116 和 21572096)、深圳市科技研发资金(Nos. JCYJ20170412152435366 和 JCYJ20170307105638498)、广东省自然科学基金(No. 2018A030310083)和深圳市诺贝尔奖科学家实验室(No. C17213101)资助项目.
} 
1). ${ }^{[11]}$ However, both of the former two types of reagents are relatively expensive and high molecular weights, while the last one is volatile, all of which render these sources uneconomical and impractical for large scale preparations. In contrast, the readily commercially available trifluoromethanesulfonyl chloride ( $\mathrm{TfCl}, \mathrm{CF}_{3} \mathrm{SO}_{2} \mathrm{Cl}$ ) is relatively inexpensive, of much lower molecular weight, and is a liquid at ambient temperature, all of which make it an ideal trifluoromethyl radical precursor. ${ }^{[12]}$ As our continuing interest in fluorine chemistry, ${ }^{[13]}$ we herein report an efficient and practical protocol for preparation of a variety of trifluoromethylated tetrahydrofurans and tetrahydropyrans using TfCl-participated 1,2-alkoxyl-trifluoromethylation of unactivated alkene under mild visible-light promoted conditions (Scheme 1). ${ }^{[14]}$
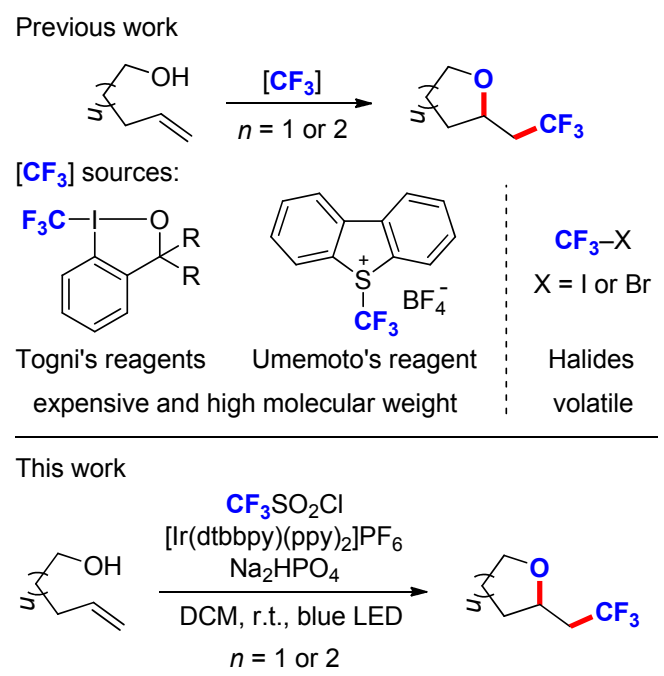

inexpensive and operatively simple $\cdot \mathrm{CF}_{3}$ source

Scheme 1 Preparation of tetrahydrofuran and tetrahydropyran via 1,2-alkoxyl-trifluoromethylation of alkene

\section{Results and discussion}

Our investigation began with 1a as a model substrate under our previously employed conditions ${ }^{[15]}$ for generation of trifluoromethyl radical: photosensitizer [ $\operatorname{Ir}(\mathrm{dtbbpy})-$ (ppy) ${ }_{2} \mathrm{PF}_{6}\left(1 \mathrm{~mol} \%\right.$ ), trifluoromethyl radical source $\mathrm{CF}_{3} \mathrm{~S}$ $\mathrm{O}_{2} \mathrm{Cl}$ (1.5 equiv.), basic additive $\mathrm{Na}_{2} \mathrm{HPO}_{4} \bullet 12 \mathrm{H}_{2} \mathrm{O}$ (2 equiv.), and solvent EtOAc under blue LED (light-emitting diode) irradiation. To our delight, the desired trifluoromethylated tetrahydrofuran $\mathbf{2 a}$ was successfully obtained, albeit in low yield (Table 1, Entry 1,36\%). Next, several common solvents (Table 1, Entries 2 11) were screened and chlorinated solvents generally performed better than others. In addition, dichloromethane (DCM) was slightly superior to 1,2-dichloroethane (Table 1, Entries 2 and 3, $70 \%$ and $68 \%$, respectively). In order to further improve the reaction efficiency, then the effect of different inorganic bases as additives was examined (Table 1, Entries 12 15). Unfortunately, all these bases were inferior to the original $\mathrm{Na}_{2} \mathrm{HPO}_{4}$. Another common photosensitizer $\mathrm{Ru}\left(\mathrm{bpy}_{3}\right) \mathrm{Cl}_{2}$ • $6 \mathrm{H}_{2} \mathrm{O}^{[14 \mathrm{c}]}$ was also examined but only low yield of desired product 2a (Table 1, Entry 16) was observed. This result was not unexpected given its relatively low reduction capability $\left(E_{\text {red }}=-0.81 \mathrm{~V}\right.$ and $-0.96 \mathrm{~V}$ vs SCE for excited states of $\mathrm{Ru}\left(\mathrm{bpy}_{3}\right)^{2+}$ and $\left[\operatorname{Ir}(\mathrm{dtbbpy})(\mathrm{ppy})_{2}\right]^{+}$, respectively). ${ }^{[14 \mathrm{e}]}$ In addition, several organic photoredox catalysts, ${ }^{[14 \mathrm{j}]}$ such as 9,10-dicyanoanthracene, Ph-Acr- $\mathrm{Me}^{+} \mathrm{ClO}_{4}^{-}$, and Mes- $\mathrm{Me}_{2} \mathrm{Acr}-\mathrm{Me}^{+} \mathrm{ClO}_{4}^{-}$, were explored (Table 1, Entries 17 19). Unfortunately, none of them provided comparable efficiency with the Ir-based photocatalyst, possibly due to different reaction mechanisms. Therefore, the optimal reaction conditions were identified as follows: [ $\operatorname{Ir}(\mathrm{dtbbpy})-$ (ppy) $\left.{ }_{2}\right] \mathrm{PF}_{6}(1 \mathrm{~mol} \%), \mathrm{CF}_{3} \mathrm{SO}_{2} \mathrm{Cl}$ (1.5 equiv.), and $\mathrm{Na}_{2} \mathrm{H}-$ $\mathrm{PO}_{4} \bullet 12 \mathrm{H}_{2} \mathrm{O}$ (2 equiv.) in dichloromethane (DCM) $(2 \mathrm{~mL})$ under blue LED irradiation, in which the desired product was obtained in $55 \%$ isolated yield on a $0.2 \mathrm{mmol}$ scale (Table 2).

With the optimized conditions in hand, the substrate scope of the current alkoxyl-trifluoromethylation reaction was subsequently explored (Table 2). Substrates bearing electron-donating or electron-withdrawing groups on the meta- or para-positions of the alkenyl phenyl rings were tolerated to afford desired trifluoromethylated tetrahydrofurans $\mathbf{2 b} \sim \mathbf{2 f}$ in $45 \% \sim 70 \%$ isolated yields. The sterically bulky substrates $\mathbf{1 g}$ and $\mathbf{1 h}$ possessing a 2-methyl substituted phenyl ring and a 1-naphtharene ring also worked well, leading to products $\mathbf{2 g}$ and $\mathbf{2 h}$, respectively. A labile benzothiophene ring in substrate $\mathbf{1 i}$ survived the reaction conditions to provide product $\mathbf{2 i}$ in $40 \%$ yield. Noteworthy is that gem-dialkyl-substituted alkene $\mathbf{1 j}$ and mono-alkylsubstituted alkenes $\mathbf{1 k}$ and $\mathbf{1 l}$ were also applicable in the reaction, giving rise to corresponding products in moderate to high yields. In addition, alkenols $\mathbf{1 m}$ and $\mathbf{1 n}$ bearing only one aryl substituent on the tether also underwent the reaction smoothly, albeit in apparently no diastereoselectivity. Most importantly, substrates $10 \sim 1$ 1q with one-carbonlonger tethers were workable under the same conditions to deliver trifluoromethylated tetrahydropyrans $\mathbf{2 0} \sim \mathbf{2 q}$ in good to excellent yields and low diastereoselectivity. Furthermore, substrate $1 \mathbf{r}$ featuring a phenyl-fused tether was also compatible with the reaction conditions to give tetrahydrobenzopyran $\mathbf{2 r}$ in moderate yield. The successful formation of trifluoromethylated tetrahydropyran under our conditions is in agreement with literature reports, which indicates that visible-light photoredox catalyzed conditions are more robust and versatile than other conditions in terms of the scope for cyclic ether products. ${ }^{[9 \sim 11,16]}$

On the basis of literature reports, we propose a possible reaction pathway for the alkoxyl-trifluoromethylation reaction (Scheme 2). First, the photosensitizer Ir(III) complex is converted to its excited state $\operatorname{Ir}(\mathrm{III})^{*}$ under blue LED irradiation, which subsequently reduces $\mathrm{TfCl}$ to provide trifluoromethyl radical and $\operatorname{Ir}(\mathrm{IV}) .{ }^{[17]} \mathrm{Next}$, the trifluoromethyl radical adds to alkene $\mathbf{1}$ to provide alkyl radical $\mathbf{I}$. This radical I is subsequently oxidized by $\operatorname{Ir}(\mathrm{IV})$ to form carbocation II, thus regenerating the initial Ir(III) complex. Finally, the desired product $\mathbf{2}$ is forged upon intramolecularly 
Table 1 Optimization of reaction conditions ${ }^{a}$
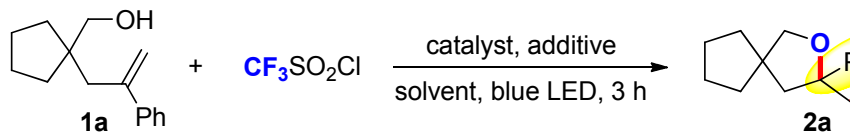

$2 a$
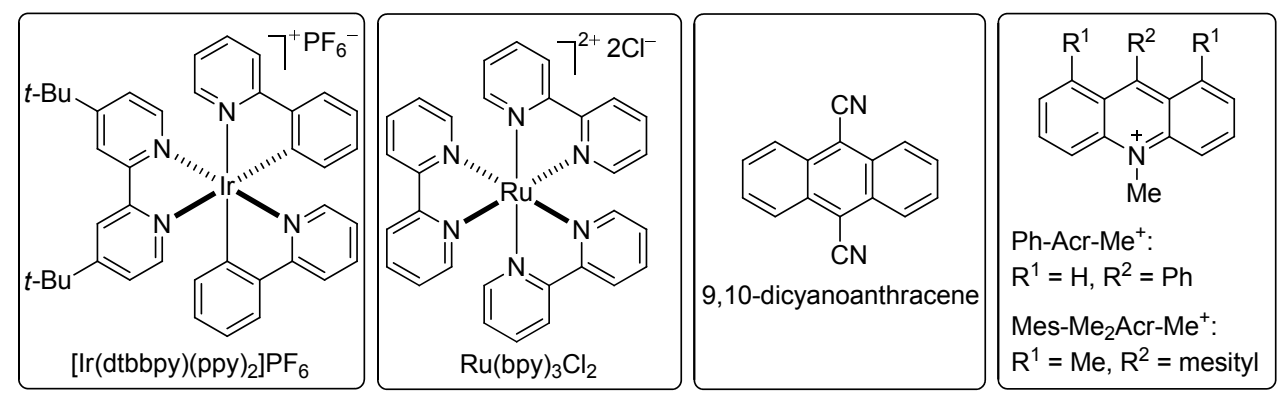

\begin{tabular}{|c|c|c|c|c|}
\hline Entry & Catalyst & Additive & Solvent & Yield/\% \\
\hline 1 & {$\left[\operatorname{Ir}(\mathrm{dtbbpy})(\mathrm{ppy})_{2}\right] \mathrm{PF}_{6}$} & $\mathrm{Na}_{2} \mathrm{HPO}_{4} \bullet 12 \mathrm{H}_{2} \mathrm{O}$ & EtOAc & 36 \\
\hline 2 & {$\left[\operatorname{Ir}(d t b b p y)(p p y)_{2}\right] P_{6}$} & $\mathrm{Na}_{2} \mathrm{HPO}_{4} \cdot 12 \mathrm{H}_{2} \mathrm{O}$ & DCM & 70 \\
\hline 3 & {$\left[\operatorname{Ir}(\mathrm{dtbbpy})(\mathrm{ppy})_{2}\right] \mathrm{PF}_{6}$} & $\mathrm{Na}_{2} \mathrm{HPO}_{4} \bullet 12 \mathrm{H}_{2} \mathrm{O}$ & DCE & 68 \\
\hline 4 & {$\left[\operatorname{Ir}(\right.$ dtbbpy $\left.)(\text { ppy })_{2}\right] \mathrm{PF}_{6}$} & $\mathrm{Na}_{2} \mathrm{HPO}_{4} \bullet 12 \mathrm{H}_{2} \mathrm{O}$ & $\mathrm{CH}_{3} \mathrm{CN}$ & 44 \\
\hline 5 & {$\left[\operatorname{Ir}(\mathrm{dtbbpy})(\mathrm{ppy})_{2}\right] \mathrm{PF}_{6}$} & $\mathrm{Na}_{2} \mathrm{HPO}_{4} \cdot 12 \mathrm{H}_{2} \mathrm{O}$ & $\mathrm{PhF}$ & 36 \\
\hline 6 & {$\left[\operatorname{Ir}(\right.$ dtbbpy $\left.)(\text { ppy })_{2}\right] \mathrm{PF}_{6}$} & $\mathrm{Na}_{2} \mathrm{HPO}_{4} \bullet 12 \mathrm{H}_{2} \mathrm{O}$ & $\mathrm{DMF}$ & 21 \\
\hline 7 & {$\left[\operatorname{Ir}(\mathrm{dtbbpy})(\mathrm{ppy})_{2}\right] \mathrm{PF}_{6}$} & $\mathrm{Na}_{2} \mathrm{HPO}_{4} \bullet 12 \mathrm{H}_{2} \mathrm{O}$ & DMSO & Messy \\
\hline 8 & {$\left[\operatorname{Ir}(\mathrm{dtbbpy})(\mathrm{ppy})_{2}\right] \mathrm{PF}_{6}$} & $\mathrm{Na}_{2} \mathrm{HPO}_{4} \bullet 12 \mathrm{H}_{2} \mathrm{O}$ & THF & 39 \\
\hline 9 & {$\left[\operatorname{Ir}(\mathrm{dtbbpy})(\mathrm{ppy})_{2}\right] \mathrm{PF}_{6}$} & $\mathrm{Na}_{2} \mathrm{HPO}_{4} \bullet 12 \mathrm{H}_{2} \mathrm{O}$ & 1,4-Dioxane & 26 \\
\hline 10 & {$\left[\operatorname{Ir}(\mathrm{dtbbpy})(\mathrm{ppy})_{2}\right] \mathrm{PF}_{6}$} & $\mathrm{Na}_{2} \mathrm{HPO}_{4} \bullet 12 \mathrm{H}_{2} \mathrm{O}$ & Cyclohexane & Messy \\
\hline 11 & {$\left[\operatorname{Ir}(\mathrm{dtbbpy})(\mathrm{ppy})_{2}\right] \mathrm{PF}_{6}$} & $\mathrm{Na}_{2} \mathrm{HPO}_{4} \bullet 12 \mathrm{H}_{2} \mathrm{O}$ & $\mathrm{MeOH}$ & 39 \\
\hline 12 & {$\left[\operatorname{Ir}(\mathrm{dtbbpy})(\mathrm{ppy})_{2}\right] \mathrm{PF}_{6}$} & $\mathrm{NaHCO}_{3}$ & DCM & 55 \\
\hline 13 & {$\left[\operatorname{Ir}(\right.$ dtbbpy $\left.)(\text { ppy })_{2}\right] \mathrm{PF}_{6}$} & $\mathrm{Na}_{2} \mathrm{CO}_{3}$ & DCM & 56 \\
\hline 14 & {$\left[\operatorname{Ir}(\right.$ dtbbpy $\left.)(\text { ppy })_{2}\right] \mathrm{PF}_{6}$} & $\mathrm{KHCO}_{3}$ & DCM & 49 \\
\hline 15 & {$\left[\operatorname{Ir}(\mathrm{dtbbpy})(\mathrm{ppy})_{2}\right] \mathrm{PF}_{6}$} & $\mathrm{~K}_{2} \mathrm{CO}_{3}$ & DCM & 31 \\
\hline 16 & $\mathrm{Ru}(\mathrm{bpy})_{3} \mathrm{Cl}_{2} \bullet 6 \mathrm{H}_{2} \mathrm{O}$ & $\mathrm{Na}_{2} \mathrm{HPO}_{4} \bullet 12 \mathrm{H}_{2} \mathrm{O}$ & DCM & 45 \\
\hline 17 & 9,10-Dicyanoanthracene & $\mathrm{Na}_{2} \mathrm{HPO}_{4} \bullet 12 \mathrm{H}_{2} \mathrm{O}$ & $\mathrm{DCM}$ & 20 \\
\hline 18 & $\mathrm{Ph}-\mathrm{Acr}-\mathrm{Me}^{+} \mathrm{ClO}_{4}^{-}$ & $\mathrm{Na}_{2} \mathrm{HPO}_{4} \cdot 12 \mathrm{H}_{2} \mathrm{O}$ & DCM & 13 \\
\hline 19 & $\mathrm{Mes}_{-} \mathrm{Me}_{2} \mathrm{Acr}-\mathrm{Me}^{+} \mathrm{ClO}_{4}^{-}$ & $\mathrm{Na}_{2} \mathrm{HPO}_{4} \bullet 12 \mathrm{H}_{2} \mathrm{O}$ & DCM & 28 \\
\hline
\end{tabular}

${ }^{a}$ Conditions: 1a $(0.1 \mathrm{mmol})$, catalyst $(1 \mathrm{~mol} \%), \mathrm{CF}_{3} \mathrm{SO}_{2} \mathrm{Cl}(1.5$ equiv.), and additive (2 equiv.) in solvent (1 $\mathrm{mL})$ under blue LED irradiation for $3 \mathrm{~h}$. Yields were based on ${ }^{19} \mathrm{~F}$ NMR spectroscopy using $\alpha, \alpha, \alpha$-trifluorotoluene as an internal standard.
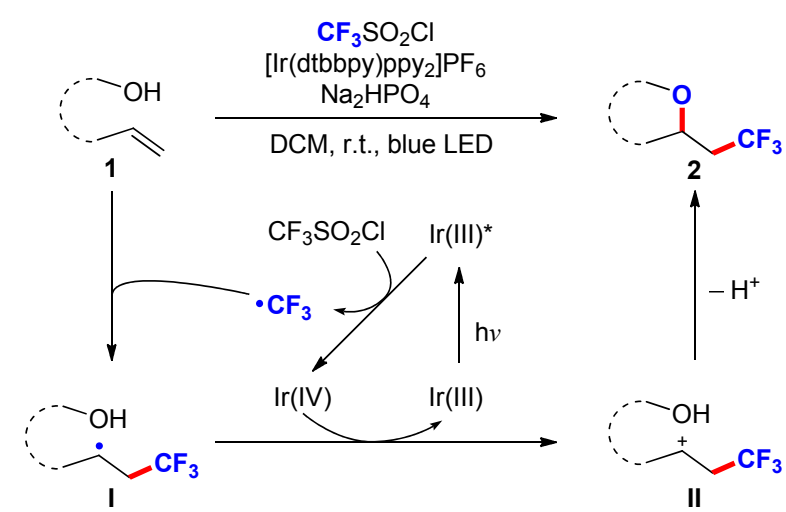

Scheme 2 Mechanistic proposal

trapping the carbocation with the alkoxyl group followed by facile deprotonation.

\section{Summary}

In sum, we have prepared a range of trifluoromethylated tetrahydrofurans and tetrahydropyrans using relative inexpensive and atom-economical trifluoromethanesulfonyl chloride as the trifluoromethyl radical source for radical 1,2-alkoxyl-trifluoromethyaltion of unactivated alkene under mild visible-light irradiation conditions. This practical protocol may find wide applications in discovering novel drugs and agrochemicals.

\section{Experimental section}

\subsection{General information}

All reactions were carried out under argon using Schlenk techniques. Unless otherwise noted, reagents were purchased at the commercial quality and used without further purification. Analytical thin layer chromatography (TLC) was performed on precoated silica gel 60 GF254 plates. Flash column chromatography was performed using Tsingdao silica gel (60, particle size $0.040 \sim 0.063 \mathrm{~mm})$. Visualization on TLC was achieved by use of UV light ( 254 $\mathrm{nm}), \mathrm{KMnO}_{4}$, or iodine stain. NMR spectra were recorded 


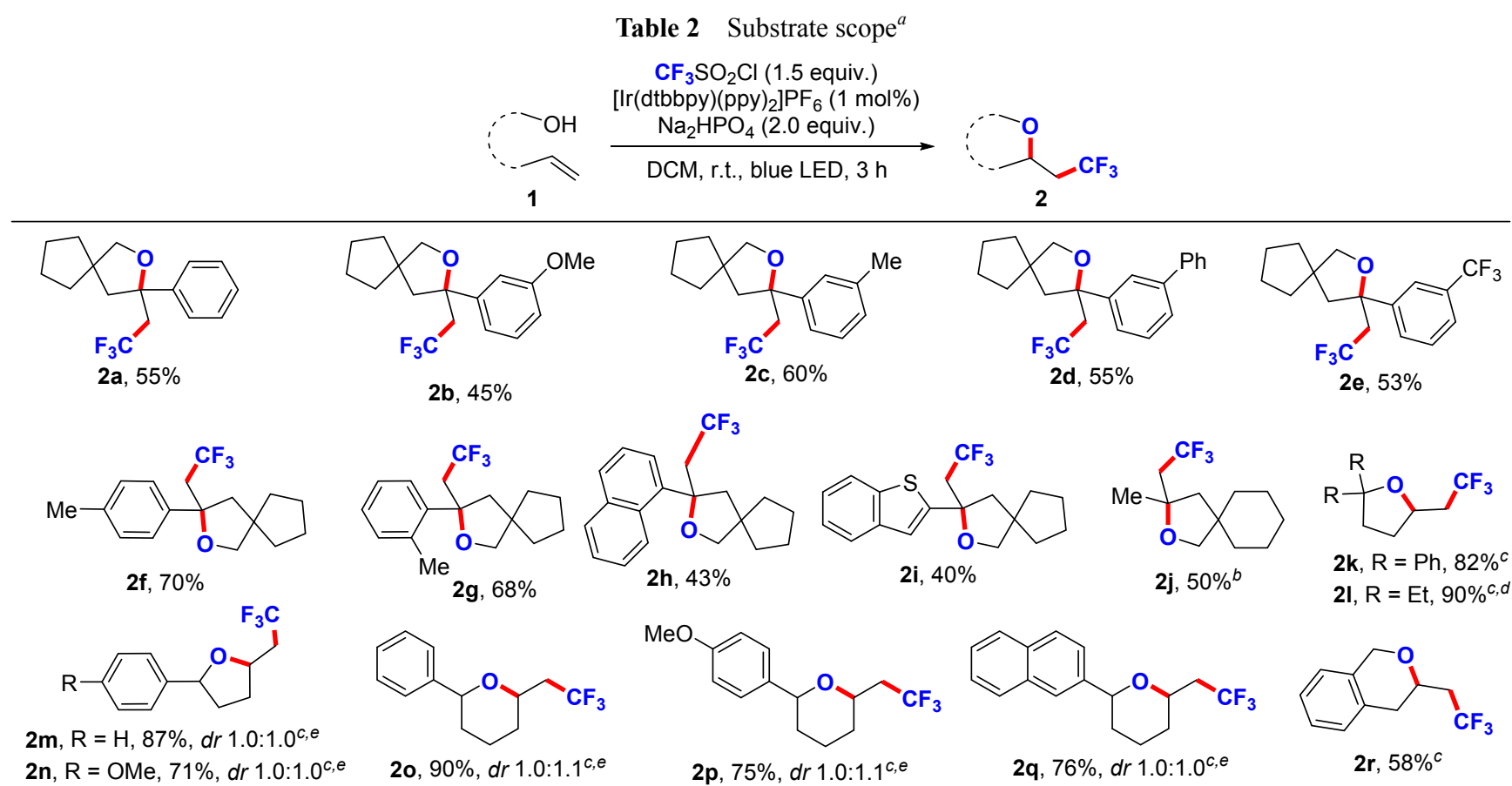

\footnotetext{
${ }^{a}$ Conditions: 1 ( $\left.0.2 \mathrm{mmol}\right),\left[\mathrm{Ir}(\mathrm{dtbbpy})(\mathrm{ppy})_{2}\right] \mathrm{PF}_{6}(1 \mathrm{~mol} \%), \mathrm{CF}_{3} \mathrm{SO}_{2} \mathrm{Cl}\left(1.5\right.$ equiv.), and $\mathrm{Na}_{2} \mathrm{HPO}_{4} \bullet 12 \mathrm{H}_{2} \mathrm{O}(2 \mathrm{equiv}$.) in DCM (2 mL) under blue LED irradiation for 3 $\mathrm{h}$, unless otherwise noted. Yields were isolated ones, unless otherwise noted. ${ }^{b}$ Reaction time: $1 \mathrm{~h} .{ }^{c}$ Reaction Time: $1.5 \mathrm{~h} .{ }^{d}$ Yield was based on ${ }^{19} \mathrm{~F}$ NMR spectroscopy using $\alpha, \alpha, \alpha$-trifluorotoluene as an internal standard. ${ }^{e}$ Diastereomeric ratio was determined based on quantitative ${ }^{13} \mathrm{C}$ NMR spectra of inseparable mixtures of both diastereomers.
}

on a Bruker DPX 400/500 spectrometers at 400/500 MHz for ${ }^{1} \mathrm{H}$ NMR, $100 / 125 \mathrm{MHz}$ for ${ }^{13} \mathrm{C} \mathrm{NMR}$, and $376 \mathrm{MHz}$ for ${ }^{19} \mathrm{~F} \mathrm{NMR}$ in $\mathrm{CDCl}_{3}$ with tetramethylsilane (TMS) as internal standard. ${ }^{19} \mathrm{~F}$ NMR spectra were recorded on a Bruker DPX $400 \mathrm{MHz}$ spectrometer $\left(\mathrm{CFCl}_{3}\right.$ as an external reference). Mass spectrometric data were obtained using a "Bruker Apex IV RTMS".

\subsection{General procedure for visible light promoted alkoxyl-trifluoromethylation of alkene}

To a 5-mL single-necked tube equipped with a magnetic stir bar were added compound 1 (0.2 mmol), [ $\operatorname{Ir}(\mathrm{dtbbpy})-$ (ppy) $)_{2} \mathrm{PF}_{6}(1.8 \mathrm{mg}, 0.002 \mathrm{mmol})$ and $\mathrm{Na}_{2} \mathrm{HPO}_{4} \cdot 12 \mathrm{H}_{2} \mathrm{O}$ (143 $\mathrm{mg}, 0.4 \mathrm{mmol}$ ). Then the reaction tube was evacuated with oil pump and back-filled with argon three times. After addition of anhydrous DCM $(2 \mathrm{~mL})$ and $\mathrm{CF}_{3} \mathrm{SO}_{2} \mathrm{Cl}(32 \mu \mathrm{L}$, $0.3 \mathrm{mmol}$ ) under argon atmosphere, the reaction tube was sealed. And the mixture was stirred under the irradiation with blue LED for appropriate time $(1 \sim 3 \mathrm{~h})$. Upon completion, the solvent was removed directly under reduced pressure to afford the crude product, which was purified by flash column chromatography to afford the desired product.

3-Phenyl-3-(2,2,2-trifluoroethyl)-2-oxaspiro[4.4]nonane (2a): ${ }^{[9 \mathrm{~d}]}$ Colourless oil, 55\% yield. ${ }^{1} \mathrm{H}$ NMR $(400 \mathrm{MHz}$, $\left.\mathrm{CDCl}_{3}\right) \delta: 7.47 \sim 7.41(\mathrm{~m}, 2 \mathrm{H}), 7.39 \sim 7.33(\mathrm{~m}, 2 \mathrm{H}), 7.31 \sim$ $7.25(\mathrm{~m}, 1 \mathrm{H}), 3.83(\mathrm{~d}, J=8.3 \mathrm{~Hz}, 1 \mathrm{H}), 3.69$ (d, $J=8.3 \mathrm{~Hz}$, $1 \mathrm{H}), 2.69$ (q, $J=10.7 \mathrm{~Hz}, 2 \mathrm{H}), 2.43$ (d, $J=12.8 \mathrm{~Hz}, 1 \mathrm{H})$, $2.32(\mathrm{~d}, J=12.7 \mathrm{~Hz}, 1 \mathrm{H}), 1.84 \sim 1.73(\mathrm{~m}, 1 \mathrm{H}), 1.71 \sim 1.43$ $(\mathrm{m}, 5 \mathrm{H}), 1.43 \sim 1.19(\mathrm{~m}, 2 \mathrm{H})$.

3-(3-Methoxyphenyl)-3-(2,2,2-trifluoroethyl)-2-oxaspiro[4.4]nonane (2b): ${ }^{[9 d]}$ Colourless oil, $45 \%$ yield. ${ }^{1} \mathrm{H}$ NMR (400 MHz, $\left.\mathrm{CDCl}_{3}\right) \delta: 7.27(\mathrm{t}, J=8.0 \mathrm{~Hz}, 1 \mathrm{H}), 7.05$ (dd, $J=2.6,1.7 \mathrm{~Hz}, 1 \mathrm{H}), 6.98(\mathrm{ddd}, J=7.7,1.8,1.0 \mathrm{~Hz}$, $1 \mathrm{H}), 6.81$ (ddd, $J=8.2,2.6,0.9 \mathrm{~Hz}, 1 \mathrm{H}), 3.87 \sim 3.80(\mathrm{~m}$, 4H), 3.70 (d, $J=8.3 \mathrm{~Hz}, 1 \mathrm{H}), 2.68$ (q, $J=10.7 \mathrm{~Hz}, 2 \mathrm{H}), 2.42$ $(\mathrm{d}, J=12.7 \mathrm{~Hz}, 1 \mathrm{H}), 2.31(\mathrm{~d}, J=12.8 \mathrm{~Hz}, 1 \mathrm{H}), 1.85 \sim 1.72$ $(\mathrm{m}, 1 \mathrm{H}), 1.72 \sim 1.44(\mathrm{~m}, 5 \mathrm{H}), 1.43 \sim 1.33(\mathrm{~m}, 1 \mathrm{H}), 1.32 \sim$ $1.21(\mathrm{~m}, 1 \mathrm{H})$.

3-(m-Tolyl)-3-(2,2,2-trifluoroethyl)-2-oxaspiro[4.4]nonane (2c): ${ }^{[9 \mathrm{~d}]}$ Colourless oil, $60 \%$ yield. ${ }^{1} \mathrm{H}$ NMR (400 $\left.\mathrm{MHz}, \mathrm{CDCl}_{3}\right) \delta: 7.25 \sim 7.15(\mathrm{~m}, 3 \mathrm{H}), 7.08 \sim 7.03(\mathrm{~m}, 1 \mathrm{H})$, $3.79(\mathrm{~d}, J=8.3 \mathrm{~Hz}, 1 \mathrm{H}), 3.66(\mathrm{~d}, J=8.3 \mathrm{~Hz}, 1 \mathrm{H}), 2.65$ (q, $J=10.7 \mathrm{~Hz}, 2 \mathrm{H}), 2.38(\mathrm{~d}, J=12.8 \mathrm{~Hz}, 1 \mathrm{H}), 2.36(\mathrm{~s}, 3 \mathrm{H})$, $2.29(\mathrm{~d}, J=12.7 \mathrm{~Hz}, 1 \mathrm{H}), 1.84 \sim 1.69(\mathrm{~m}, 1 \mathrm{H}), 1.70 \sim 1.42$ $(\mathrm{m}, 5 \mathrm{H}), 1.39 \sim 1.29(\mathrm{~m}, 1 \mathrm{H}), 1.29 \sim 1.18(\mathrm{~m}, 1 \mathrm{H})$.

3-([1,1'-Biphenyl]-3-yl)-3-(2,2,2-trifluoroethyl)-2oxaspiro[4.4]nonane (2d): ${ }^{[9 \mathrm{~d}]}$ Colourless oil, $55 \%$ yield. ${ }^{1} \mathrm{H}$ NMR (400 MHz, $\left.\mathrm{CDCl}_{3}\right) \delta: 7.67 \sim 7.65(\mathrm{~m}, 1 \mathrm{H}), 7.60(\mathrm{~d}$, $J=7.4 \mathrm{~Hz}, 2 \mathrm{H}), 7.51 \sim 7.30(\mathrm{~m}, 6 \mathrm{H}), 3.82(\mathrm{~d}, J=8.3 \mathrm{~Hz}$, $1 \mathrm{H}), 3.69(\mathrm{~d}, J=8.4 \mathrm{~Hz}, 1 \mathrm{H}), 2.71(\mathrm{q}, J=10.7 \mathrm{~Hz}, 2 \mathrm{H}), 2.45$ $(\mathrm{d}, J=12.7 \mathrm{~Hz}, 1 \mathrm{H}), 2.34(\mathrm{~d}, J=12.8 \mathrm{~Hz}, 1 \mathrm{H}), 1.84 \sim 1.70$ $(\mathrm{m}, 1 \mathrm{H}), 1.70 \sim 1.40(\mathrm{~m}, 5 \mathrm{H}), 1.41 \sim 1.29(\mathrm{~m}, 1 \mathrm{H}), 1.33 \sim$ $1.19(\mathrm{~m}, 1 \mathrm{H})$.

3-(2,2,2-Trifluoroethyl)-3-(3-(trifluoromethyl)phenyl)2-oxaspiro[4.4]nonane (2e): ${ }^{[9 \mathrm{~d}]}$ Colourless oil, 53\% yield. ${ }^{1} \mathrm{H}$ NMR $\left(400 \mathrm{MHz}, \mathrm{CDCl}_{3}\right) \delta: 7.72(\mathrm{~s}, 1 \mathrm{H}), 7.62(\mathrm{~d}, J=7.8$ $\mathrm{Hz}, 1 \mathrm{H}), 7.54$ (d, $J=7.8 \mathrm{~Hz}, 1 \mathrm{H}), 7.48$ (t, $J=7.7 \mathrm{~Hz}, 1 \mathrm{H})$, $3.84(\mathrm{~d}, J=8.4 \mathrm{~Hz}, 1 \mathrm{H}), 3.69$ (d, $J=8.4 \mathrm{~Hz}, 1 \mathrm{H}), 2.79 \sim$ $2.62(\mathrm{~m}, 2 \mathrm{H}), 2.43 \sim 2.33(\mathrm{~m}, 2 \mathrm{H}), 1.86 \sim 1.73(\mathrm{~m}, 1 \mathrm{H})$, $1.73 \sim 1.43(\mathrm{~m}, 5 \mathrm{H}), 1.40 \sim 1.16(\mathrm{~m}, 2 \mathrm{H})$.

3-( $p$-Tolyl)-3-(2,2,2-trifluoroethyl)-2-oxaspiro[4.4]nonane (2f) ${ }^{[9 \mathrm{~d}]}$ Colourless oil, $70 \%$ yield. ${ }^{1} \mathrm{H}$ NMR (400 $\left.\mathrm{MHz}, \mathrm{CDCl}_{3}\right) \delta: 7.29(\mathrm{~d}, J=7.9 \mathrm{~Hz}, 2 \mathrm{H}), 7.13(\mathrm{~d}, J=7.8$ 
$\mathrm{Hz}, 2 \mathrm{H}), 3.78$ (d, $J=8.3 \mathrm{~Hz}, 1 \mathrm{H}), 3.64$ (d, $J=8.3 \mathrm{~Hz}, 1 \mathrm{H})$, 2.65 (q, $J=10.8 \mathrm{~Hz}, 2 \mathrm{H}), 2.38(\mathrm{~d}, J=12.7 \mathrm{~Hz}, 1 \mathrm{H}), 2.33$ (s, $3 \mathrm{H}), 2.27(\mathrm{~d}, J=12.8 \mathrm{~Hz}, 1 \mathrm{H}), 1.84 \sim 1.68(\mathrm{~m}, 1 \mathrm{H}), 1.70 \sim$ $1.39(\mathrm{~m}, 5 \mathrm{H}), 1.40 \sim 1.26(\mathrm{~m}, 1 \mathrm{H}), 1.29 \sim 1.17(\mathrm{~m}, 1 \mathrm{H})$.

3-(o-Tolyl)-3-(2,2,2-trifluoroethyl)-2-oxaspiro[4.4]nonane $(\mathbf{2 g}):{ }^{[9 \mathrm{~d}]}$ Colourless oil, $68 \%$ yield. ${ }^{1} \mathrm{H}$ NMR (400 $\left.\mathrm{MHz}, \mathrm{CDCl}_{3}\right) \delta: 7.74 \sim 7.63(\mathrm{~m}, 1 \mathrm{H}), 7.23 \sim 7.07(\mathrm{~m}, 3 \mathrm{H})$, $3.79(\mathrm{~d}, J=8.4 \mathrm{~Hz}, 1 \mathrm{H}), 3.60(\mathrm{~d}, J=8.4 \mathrm{~Hz}, 1 \mathrm{H}), 2.93 \sim$ $2.63(\mathrm{~m}, 2 \mathrm{H}), 2.40 \sim 2.31(\mathrm{~m}, 5 \mathrm{H}), 1.90 \sim 1.78(\mathrm{~m}, 1 \mathrm{H})$, $1.75 \sim 1.44(\mathrm{~m}, 5 \mathrm{H}), 1.47 \sim 1.22(\mathrm{~m}, 2 \mathrm{H})$.

3-(Naphthalen-1-yl)-3-(2,2,2-trifluoroethyl)-2-oxaspiro[4.4]nonane (2h): Colourless oil, $43 \%$ yield. ${ }^{1} \mathrm{H}$ NMR (400 $\left.\mathrm{MHz}, \mathrm{CDCl}_{3}\right) \delta: 7.96 \sim 7.81(\mathrm{~m}, 3 \mathrm{H}), 7.78(\mathrm{~d}, J=8.2 \mathrm{~Hz}$, $1 \mathrm{H}), 7.54 \sim 7.43(\mathrm{~m}, 3 \mathrm{H}), 3.88(\mathrm{~d}, J=8.4 \mathrm{~Hz}, 1 \mathrm{H}), 3.70(\mathrm{~d}$, $J=8.3 \mathrm{~Hz}, 1 \mathrm{H}), 3.16 \sim 2.89(\mathrm{~m}, 2 \mathrm{H}), 2.71 \sim 2.58(\mathrm{~m}, 2 \mathrm{H})$, $1.96 \sim 1.83(\mathrm{~m}, 1 \mathrm{H}), 1.77 \sim 1.53(\mathrm{~m}, 5 \mathrm{H}), 1.57 \sim 1.43(\mathrm{~m}$, $1 \mathrm{H}), 1.45 \sim 1.33(\mathrm{~m}, 1 \mathrm{H}) ;{ }^{13} \mathrm{C}$ NMR $\left(100 \mathrm{MHz}, \mathrm{CDCl}_{3}\right) \delta$ : $140.75,134.87,129.82,129.64,128.53,125.82,125.64$ (q, $J=278.9 \mathrm{~Hz}), 125.41,125.06,124.75,123.56,83.30 \sim$ $83.05(\mathrm{~m}), 77.79,53.30 \sim 52.90(\mathrm{~m}), 51.59,45.06(\mathrm{q}, J=$ $26.0 \mathrm{~Hz}), 38.33$, 36.76, 24.95, 24.77; ${ }^{19} \mathrm{~F}$ NMR (376 MHz, $\mathrm{CDCl}_{3}$ ) $\delta$ : -60.54 ; HRMS (ESI) calcd for $\mathrm{C}_{20} \mathrm{H}_{22} \mathrm{~F}_{3} \mathrm{O}$ $[\mathrm{M}+\mathrm{H}]^{+}$335.1617, found 335.1613.

3-(Benzo[b]thiophen-3-yl)-3-(2,2,2-trifluoroethyl)-2oxaspiro[4.4]nonane (2i): Colourless oil, $40 \%$ yield. ${ }^{1} \mathrm{H}$ NMR $\left(400 \mathrm{MHz}, \mathrm{CDCl}_{3}\right) \delta: 7.93 \sim 7.87(\mathrm{~m}, 1 \mathrm{H}), 7.85 \sim$ $7.78(\mathrm{~m}, 1 \mathrm{H}), 7.50(\mathrm{~s}, 1 \mathrm{H}), 7.46 \sim 7.33(\mathrm{~m}, 2 \mathrm{H}), 3.86(\mathrm{~d}$, $J=8.4 \mathrm{~Hz}, 1 \mathrm{H}), 3.74(\mathrm{~d}, J=8.3 \mathrm{~Hz}, 1 \mathrm{H}), 3.00 \sim 2.85(\mathrm{~m}$, 2H), 2.61 (d, $J=12.8 \mathrm{~Hz}, 1 \mathrm{H}), 2.42$ (d, $J=12.7 \mathrm{~Hz}, 1 \mathrm{H})$, $1.93 \sim 1.79(\mathrm{~m}, 1 \mathrm{H}), 1.78 \sim 1.43(\mathrm{~m}, 5 \mathrm{H}), 1.43 \sim 1.25(\mathrm{~m}$, $2 \mathrm{H}) ;{ }^{13} \mathrm{C} \mathrm{NMR}\left(100 \mathrm{MHz}, \mathrm{CDCl}_{3}\right) \delta: 141.79,139.56$, $136.51,124.13,124.11,123.41,122.93,122.90,82.35$ (q, $J=2.0 \mathrm{~Hz}), 78.54,51.38,50.78,44.17(\mathrm{q}, J=26.1 \mathrm{~Hz})$, $38.28,37.31,24.84,24.76 ;{ }^{19} \mathrm{~F}$ NMR (376 MHz, $\left.\mathrm{CDCl}_{3}\right) \delta$ : -61.01; HRMS (ESI) calcd for $\mathrm{C}_{18} \mathrm{H}_{20} \mathrm{~F}_{3} \mathrm{OS}[\mathrm{M}+\mathrm{H}]^{+}$ 341.1181, found 341.1190.

3-Methyl-3-(2,2,2-trifluoroethyl)-2-oxaspiro[4.5]decane (2j): Colourless oil, 50\% yield. ${ }^{1} \mathrm{H} \mathrm{NMR}\left(500 \mathrm{MHz}, \mathrm{CDCl}_{3}\right)$ $\delta: 3.79(\mathrm{dd}, J=11.9,3.9 \mathrm{~Hz}, 1 \mathrm{H}), 3.68(\mathrm{dd}, J=11.9,5.1 \mathrm{~Hz}$, $1 \mathrm{H}), 2.87 \sim 2.72(\mathrm{~m}, 2 \mathrm{H}), 2.06 \sim 1.96(\mathrm{~m}, 2 \mathrm{H}), 1.85 \sim 1.81$ $(\mathrm{m}, 3 \mathrm{H}), 1.58 \sim 1.32(\mathrm{~m}, 10 \mathrm{H}) ;{ }^{13} \mathrm{C}$ NMR $(125 \mathrm{MHz}$, $\left.\mathrm{CDCl}_{3}\right) \delta: 125.22(\mathrm{q}, J=279.0 \mathrm{~Hz}), 68.76 \sim 68.65(\mathrm{~m})$, 66.02 (br s), 49.91 (br s), 49.44 (q, $J=27.2 \mathrm{~Hz}$ ), 39.69, $35.06,34.46,31.48,26.20,21.66,21.60 ;{ }^{19} \mathrm{~F}$ NMR $(376$ $\left.\mathrm{MHz}, \mathrm{CDCl}_{3}\right) \quad \delta$ : -59.79 ; HRMS (ESI) calcd for $\mathrm{C}_{12} \mathrm{H}_{20} \mathrm{~F}_{3} \mathrm{O}[\mathrm{M}+\mathrm{H}]^{+}$237.1461, found 237.1459.

4,4-Diphenyl-2-(2,2,2-trifluoroethyl)tetrahydrofuran (2k): Colourless oil, 82\% yield. ${ }^{1} \mathrm{H}$ NMR (400 MHz, $\left.\mathrm{CDCl}_{3}\right) \delta: 7.49 \sim 7.41(\mathrm{~m}, 4 \mathrm{H}), 7.40 \sim 7.33(\mathrm{~m}, 4 \mathrm{H}), 7.32 \sim$ $7.25(\mathrm{~m}, 2 \mathrm{H}), 4.26 \sim 4.07(\mathrm{~m}, 1 \mathrm{H}), 2.74 \sim 2.36(\mathrm{~m}, 4 \mathrm{H})$, $2.04 \sim 1.85(\mathrm{~m}, 1 \mathrm{H}), 1.83 \sim 1.68(\mathrm{~m}, 1 \mathrm{H}) ;{ }^{13} \mathrm{C}$ NMR $(100$ $\left.\mathrm{MHz}, \mathrm{CDCl}_{3}\right) \delta: 146.76,146.29,128.49,128.47,127.33$, 127.26, 126.09, 126.02, 125.31 (d, $J=277.5 \mathrm{~Hz}), 77.89$, 54.78 (q, $J=2.9 \mathrm{~Hz}), 42.68$ (q, $J=28.6 \mathrm{~Hz}), 38.33,32.87$; ${ }^{19} \mathrm{~F}$ NMR $\left(376 \mathrm{MHz}, \mathrm{CDCl}_{3}\right) \delta$ : -63.88 ; HRMS (ESI) calcd for $\mathrm{C}_{18} \mathrm{H}_{18} \mathrm{~F}_{3} \mathrm{O}[\mathrm{M}+\mathrm{H}]^{+} 307.1304$, found 307.1294.

4,4-Diethyl-2-(2,2,2-trifluoroethyl)tetrahydrofuran (21):
Colourless oil, 90\% yield (based on ${ }^{19} \mathrm{~F}$ NMR). ${ }^{1} \mathrm{H}$ NMR $\left(400 \mathrm{MHz}, \mathrm{CDCl}_{3}\right) \delta: 4.21 \sim 4.07(\mathrm{~m}, 1 \mathrm{H}), 2.74 \sim 2.50(\mathrm{~m}$, $2 \mathrm{H}), 2.01 \sim 1.88(\mathrm{~m}, 1 \mathrm{H}), 1.85 \sim 1.68(\mathrm{~m}, 2 \mathrm{H}), 1.59 \sim 1.43$ $(\mathrm{m}, 5 \mathrm{H}), 0.98 \sim 0.83(\mathrm{~m}, 6 \mathrm{H}) ;{ }^{13} \mathrm{C} \mathrm{NMR}\left(100 \mathrm{MHz}, \mathrm{CDCl}_{3}\right)$ $\delta: 125.39$ (q, $J=277.6 \mathrm{~Hz}), 74.35,54.96(\mathrm{q}, J=3.2 \mathrm{~Hz})$, 42.59 (q, $J=28.4 \mathrm{~Hz}), 34.50,32.30,31.31,30.86,7.92$, 7.76; ${ }^{19} \mathrm{~F}$ NMR $\left(376 \mathrm{MHz}, \mathrm{CDCl}_{3}\right) \delta:-63.87$; HRMS (ESI) calcd for $\mathrm{C}_{12} \mathrm{H}_{22} \mathrm{~F}_{3} \mathrm{O}[\mathrm{M}+\mathrm{H}]^{+}$211.1304, found 211.1326.

2-Phenyl-5-(2,2,2-trifluoroethyl)tetrahydrofuran (2m): Colourless oil, $87 \%$ yield, $d r 1.0: 1.0$ (based on quantitative ${ }^{13} \mathrm{C}$ NMR). ${ }^{1} \mathrm{H}$ NMR (400 MHz, $\left.\mathrm{CDCl}_{3}\right) \delta: 7.40 \sim 7.26$ $(\mathrm{m}, 5 \mathrm{H}+5 \mathrm{H}), 4.80 \sim 4.64(\mathrm{~m}, 1 \mathrm{H}+1 \mathrm{H}), 4.22 \sim 4.07(\mathrm{~m}$, $1 \mathrm{H}+1 \mathrm{H}), 2.69 \sim 2.43(\mathrm{~m}, 2 \mathrm{H}+2 \mathrm{H}), 2.11 \sim 1.95(\mathrm{~m}, 2 \mathrm{H}+$ $2 \mathrm{H}), 1.95 \sim 1.81(\mathrm{~m}, 2 \mathrm{H}+1 \mathrm{H}), 1.79 \sim 1.67(\mathrm{~m}, 1 \mathrm{H}) ;{ }^{13} \mathrm{C}$ NMR $\left(126 \mathrm{MHz}, \mathrm{CDCl}_{3}\right) \delta: 144.24,144.17,128.79,128.76$, $128.66 \sim 122.01(\mathrm{~m}, 1 \mathrm{C}+1 \mathrm{C}), 128.04,127.98,125.88$, $125.82,74.18,73.59,54.37$ (q, $J=3.3 \mathrm{~Hz}), 53.97$ (q, $J=3.2$ $\mathrm{Hz}), 42.93 \sim 42.19(\mathrm{~m}, 1 \mathrm{C}+1 \mathrm{C}), 35.46,35.15,34.71$, 34.26; ${ }^{19} \mathrm{~F}$ NMR $\left(376 \mathrm{MHz}, \mathrm{CDCl}_{3}\right) \delta$ : - 63.83; HRMS (ESI) calcd for $\mathrm{C}_{12} \mathrm{H}_{14} \mathrm{~F}_{3} \mathrm{O}[\mathrm{M}+\mathrm{H}]^{+}$231.0991, found 231.0993.

2-(4-Methoxyphenyl)-5-(2,2,2-trifluoroethyl)tetrahydrofuran (2n): Colourless oil, 71\% yield, $d r 1.0: 1.0$ (based on quantitative $\left.{ }^{13} \mathrm{C} \mathrm{NMR}\right) .{ }^{1} \mathrm{H}$ NMR (400 $\left.\mathrm{MHz}, \mathrm{CDCl}_{3}\right) \delta$ : $7.30 \sim 7.21(\mathrm{~m}, 2 \mathrm{H}+2 \mathrm{H}), 6.93 \sim 6.84(\mathrm{~m}, 2 \mathrm{H}+2 \mathrm{H}), 4.70 \sim$ $4.59(\mathrm{~m}, 1 \mathrm{H}+1 \mathrm{H}), 4.23 \sim 4.06(\mathrm{~m}, 1 \mathrm{H}+1 \mathrm{H}), 3.80(\mathrm{~s}, 3 \mathrm{H}+$ $3 \mathrm{H}), 2.69 \sim 2.41(\mathrm{~m}, 2 \mathrm{H}+2 \mathrm{H}), 2.14 \sim 1.77(\mathrm{~m}, 3 \mathrm{H}+3 \mathrm{H})$, $1.76 \sim 1.62(\mathrm{~m}, 1 \mathrm{H}+1 \mathrm{H}) ;{ }^{13} \mathrm{C} \mathrm{NMR}\left(125 \mathrm{MHz}, \mathrm{CDCl}_{3}\right) \delta$ : $159.37,159.33,136.37,136.29,128.67 \sim 122.01(\mathrm{~m}, 1 \mathrm{C}+$ 1C), 127.17, 127.10, 114.11, 114.09, 73.77, 73.23, 54.37 (q, $J=3.3 \mathrm{~Hz}), 53.99(\mathrm{q}, J=3.2 \mathrm{~Hz}), 42.93 \sim 42.18(\mathrm{~m}, 1 \mathrm{C}+$ 1C), 35.36, 35.08, 34.79, 34.38; ${ }^{19} \mathrm{~F}$ NMR (376 MHz, $\left.\mathrm{CDCl}_{3}\right) \delta$ : -63.83 ; HRMS (ESI) calcd for $\mathrm{C}_{13} \mathrm{H}_{16} \mathrm{~F}_{3} \mathrm{O}_{2}$ $[\mathrm{M}+\mathrm{H}]^{+}$261.1097, found 261.1088.

2-Phenyl-6-(2,2,2-trifluoroethyl)tetrahydro-2 $\mathrm{H}$-pyran (2o): Colourless oil, 90\% yield, $d r 1.0: 1.1$ (based on quantitative $\left.{ }^{13} \mathrm{C} \mathrm{NMR}\right) .{ }^{1} \mathrm{H} \mathrm{NMR}\left(400 \mathrm{MHz}, \mathrm{CDCl}_{3}\right) \delta$ : $7.47 \sim 7.27(\mathrm{~m}, 5 \mathrm{H}+5 \mathrm{H}), 4.76 \sim 4.65(\mathrm{~m}, 1 \mathrm{H}+1 \mathrm{H}), 4.19 \sim$ $4.05(\mathrm{~m}, 1 \mathrm{H}+1 \mathrm{H}), 2.74 \sim 2.44(\mathrm{~m}, 2 \mathrm{H}+2 \mathrm{H}), 1.98 \sim 1.53$ $(\mathrm{m}, 5 \mathrm{H}+6 \mathrm{H}), 1.54 \sim 1.38(\mathrm{~m}, 1 \mathrm{H}) ;{ }^{13} \mathrm{C} \mathrm{NMR}(125 \mathrm{MHz}$, $\left.\mathrm{CDCl}_{3}\right) \delta: 144.58(1.1 \mathrm{C}), 144.56(1 \mathrm{C}), 128.67(2.2 \mathrm{C}+2 \mathrm{C})$, 127.85 (1.1C), 127.83 (1C), 125.94 (2.2C), 125.90 (2C), $125.36(\mathrm{~d}, J=277.5 \mathrm{~Hz}, 1.1 \mathrm{C}+1 \mathrm{C}), 74.47$ (1.1C), 74.38 (1C), $54.14 \sim 54.06(\mathrm{~m}, 1.1 \mathrm{C}+1 \mathrm{C}), 42.83 \sim 42.13(\mathrm{~m}, 1.1 \mathrm{C}$ +1C), 38.20 (1C), 38.18 (1.1C), 37.99 (1C), 37.95 (1.1C), 22.52 (1C), 22.49 (1.1C); ${ }^{19} \mathrm{~F}$ NMR (376 $\left.\mathrm{MHz}, \mathrm{CDCl}_{3}\right) \delta$ : - 63.78; HRMS (ESI) calcd for $\mathrm{C}_{13} \mathrm{H}_{16} \mathrm{~F}_{3} \mathrm{O}[\mathrm{M}+\mathrm{H}]^{+}$ 245.1148 , found 245.1139 .

2-(4-Methoxyphenyl)-6-(2,2,2-trifluoroethyl)tetrahydro$2 H$-pyran (2p): Colourless oil, $75 \%$ yield, $d r 1.0: 1.0$ (based on quantitative ${ }^{13} \mathrm{C}$ NMR). ${ }^{1} \mathrm{H}$ NMR (400 MHz, $\left.\mathrm{CDCl}_{3}\right) \delta: 7.28(\mathrm{~d}, J=8.5 \mathrm{~Hz}, 2 \mathrm{H}+2 \mathrm{H}), 6.91(\mathrm{~d}, J=8.7 \mathrm{~Hz}$, $2 \mathrm{H}+2 \mathrm{H}), 4.68 \sim 4.57(\mathrm{~m}, 1 \mathrm{H}+1 \mathrm{H}), 4.17 \sim 4.04(\mathrm{~m}, 1 \mathrm{H}+$ $1 \mathrm{H}), 3.83(\mathrm{~s}, 3 \mathrm{H}+3 \mathrm{H}), 2.72 \sim 2.43(\mathrm{~m}, 2 \mathrm{H}+2 \mathrm{H}), 1.94 \sim$ $1.51(\mathrm{~m}, 5 \mathrm{H}+6 \mathrm{H}), 1.51 \sim 1.36(\mathrm{~m}, 1 \mathrm{H}) ;{ }^{13} \mathrm{C}$ NMR $(125$ $\left.\mathrm{MHz}, \mathrm{CDCl}_{3}\right) \delta: 159.24$ (1.1C), 159.23 (1C), 136.73 (1C), 
136.70 (1.1C), 127.21 (2.2C), 127.17 (2C), 125.37 (d, $J=$ $277.4 \mathrm{~Hz}, 1.1 \mathrm{C}+1 \mathrm{C}), 114.02(2.2 \mathrm{C}+2 \mathrm{C}), 74.07(1 \mathrm{C})$, $74.00(1.1 \mathrm{C}), 55.39(1.1 \mathrm{C}+1 \mathrm{C}), 54.16 \sim 54.07(\mathrm{~m}, 1.1 \mathrm{C}+$ $1 \mathrm{C}), 42.84 \sim 42.13(\mathrm{~m}, 1.1 \mathrm{C}+1 \mathrm{C}), 38.11(1 \mathrm{C}), 38.09$ (1.1C), 38.00 (1.1C), 37.97 (1C), 22.58 (1C), 22.55 (1.1C); ${ }^{19} \mathrm{~F}$ NMR $\left(376 \mathrm{MHz}, \mathrm{CDCl}_{3}\right) \delta$ : -63.81 ; HRMS (ESI) calcd for $\mathrm{C}_{14} \mathrm{H}_{18} \mathrm{~F}_{3} \mathrm{O}_{2}[\mathrm{M}+\mathrm{H}]^{+}$275.1253, found 245.1244.

2-(Naphthalen-2-yl)-6-(2,2,2-trifluoroethyl)tetrahydro-2 $H$-pyran (2q): Colourless oil, 76\% yield, $d r 1.0: 1.0$ (based on quantitative ${ }^{13} \mathrm{C}$ NMR). ${ }^{1} \mathrm{H}$ NMR (400 MHz, $\left.\mathrm{CDCl}_{3}\right) \delta$ : $7.90 \sim 7.83(\mathrm{~m}, 3 \mathrm{H}+3 \mathrm{H}), 7.80(\mathrm{~s}, 2 \mathrm{H}), 7.57 \sim 7.50(\mathrm{~m}, 2 \mathrm{H}$ $+3 \mathrm{H}), 7.48(\mathrm{t}, J=2.0 \mathrm{~Hz}, 1 \mathrm{H}), 4.91 \sim 4.82(\mathrm{~m}, 1 \mathrm{H}+1 \mathrm{H})$, $4.18 \sim 4.05(\mathrm{~m}, 1 \mathrm{H}+1 \mathrm{H}), 2.72 \sim 2.43(\mathrm{~m}, 2 \mathrm{H}+2 \mathrm{H}) ; 2.01 \sim$ $1.58(\mathrm{~m}, 5 \mathrm{H}+6 \mathrm{H}), 1.56 \sim 1.42(\mathrm{~m}, 1 \mathrm{H}) ;{ }^{13} \mathrm{C}$ NMR $(126$ $\left.\mathrm{MHz}, \mathrm{CDCl}_{3}\right) \delta: 141.90,141.87,133.35(1 \mathrm{C}+1 \mathrm{C}), 133.14$, $133.13,128.56,128.54,128.03(1 \mathrm{C}+1 \mathrm{C}), 127.82(1 \mathrm{C}+$ $1 \mathrm{C}), 126.38(1 \mathrm{C}+1 \mathrm{C}), 126.06(1 \mathrm{C}+1 \mathrm{C}), 125.36(\mathrm{q}, J=$ $277.5 \mathrm{~Hz}, 1 \mathrm{C}+1 \mathrm{C}), 124.74,124.68,123.99(1 \mathrm{C}+1 \mathrm{C})$, $74.59,74.49,54.14 \sim 54.05(\mathrm{~m}, 1 \mathrm{C}+1 \mathrm{C}), 42.82 \sim 42.12$ $(\mathrm{m}, 1 \mathrm{C}+1 \mathrm{C}), 38.06(1 \mathrm{C}+1 \mathrm{C}), 37.99,37.96,22.54,22.49$; ${ }^{19} \mathrm{~F}$ NMR $\left(376 \mathrm{MHz}, \mathrm{CDCl}_{3}\right) \delta$ : -63.76 ; HRMS (ESI) calcd for $\mathrm{C}_{17} \mathrm{H}_{18} \mathrm{~F}_{3} \mathrm{O}[\mathrm{M}+\mathrm{H}]^{+}$295.1304, found 295.1300.

3-(2,2,2-Trifluoroethyl)isochromane (2r): Colourless oil, $58 \%$ yield. ${ }^{1} \mathrm{H} \mathrm{NMR}\left(400 \mathrm{MHz}, \mathrm{CDCl}_{3}\right) \delta: 7.44 \sim 7.39(\mathrm{~m}$, $1 \mathrm{H}), 7.38 \sim 7.30(\mathrm{~m}, 2 \mathrm{H}), 7.30 \sim 7.26(\mathrm{~m}, 1 \mathrm{H}), 4.81 \sim 4.69$ $(\mathrm{m}, 2 \mathrm{H}), 4.53 \sim 4.42(\mathrm{~m}, 1 \mathrm{H}), 3.29(\mathrm{dd}, J=14.5,6.0 \mathrm{~Hz}$, $1 \mathrm{H}), 3.21(\mathrm{dd}, J=14.5,8.6 \mathrm{~Hz}, 1 \mathrm{H}), 2.77 \sim 2.61(\mathrm{~m}, 2 \mathrm{H})$; ${ }^{13} \mathrm{C}$ NMR $\left(125 \mathrm{MHz}, \mathrm{CDCl}_{3}\right) \delta: 138.94,135.54,130.81$, $129.50,128.52,127.85,125.44$ (q, $J=277.6 \mathrm{~Hz}), 63.61$, $54.51(\mathrm{q}, J=3.1 \mathrm{~Hz}), 42.05(\mathrm{q}, J=28.6 \mathrm{~Hz}), 41.10 ;{ }^{19} \mathrm{~F}$ NMR (376 MHz, $\left.\mathrm{CDCl}_{3}\right) \delta$ : -63.51; HRMS (ESI) calcd for $\mathrm{C}_{11} \mathrm{H}_{12} \mathrm{~F}_{3} \mathrm{O}[\mathrm{M}+\mathrm{H}]^{+}$217.0835, found 217.0834.

Supporting Information ${ }^{1} \mathrm{H}$ NMR and ${ }^{13} \mathrm{C}$ NMR spectra of the products. The Supporting Information is available free of charge via the Internet at http://sioc-journal.cn.

\section{References}

[1] (a) Guéret, S. M.; Brimble, M. A. Nat. Prod. Rep. 2010, 27, 1350. (b) Lorente, A.; Lamariano-Merketegi, J.; Albericio, F.; Álvarez, M. Chem. Rev. 2013, 113, 4567.

(c) Xu, Y.; Wang, W.; Yang, J.; Li, X.; Meng, Q. Chin. J. Org. Chem. 2016, 36, 724 (in Chinese).

(徐阳荣, 王文智, 杨静静, 李新利, 孟庆国, 有机化学, 2016, 36, 724.)

[2] (a) Boivin, T. L. B. Tetrahedron 1987, 43, 3309.

(b) Nasir, N. M.; Ermanis, K.; Clarke, P. A. Org. Biomol. Chem. 2014, 12, 3323.

(c) Tikad, A.; Delbrouck, J. A.; Vincent, S. P. Chem.-Eur. J. 2016, 22, 9456.

[3] (a) Müller, K.; Faeh, C.; Diederich, F. Science 2007, 317, 1881. (b) Gillis, E. P.; Eastman, K. J.; Hill, M. D.; Donnelly, D. J.; Meanwell, N. A. J. Med. Chem. 2015, 58, 8315.

(c) Zhou, Y.; Wang, J.; Gu, Z.; Wang, S.; Zhu, W.; Aceña, J. L.; Soloshonok, V. A.; Izawa, K.; Liu, H. Chem. Rev. 2016, 116, 422. (d) Meanwell, N. A. J. Med. Chem. 2018, 61, 5822.

[4] (a) Jeschke, P. ChemBioChem 2004, 5, 570.

(b) Jeschke, P. Pest Manage. Sci. 2010, 66, 10.

(c) Fujiwara, T.; O'Hagan, D. J. Fluorine Chem. 2014, 167, 16.

[5] Ojima, I. Fluorine in Medicinal Chemistry and Chemical Biology,
Blackwell Publishing Ltd, West Sussex, 2009

[6] (a) McClinton, M. A.; McClinton, D. A. Tetrahedron 1992, 48, 6555.

(b) Dolbier, W. R. Chem. Rev. 1996, 96, 1557.

(c) Furuya, T.; Kamlet, A. S.; Ritter, T. Nature 2011, 473, 470.

(d) Nie, J.; Guo, H.-C.; Cahard, D.; Ma, J.-A. Chem. Rev. 2011, 111, 455.

(e) Tomashenko, O. A.; Grushin, V. V. Chem. Rev. 2011, 111, 4475. (f) Prakash, G. K. S.; Wang, F. In Organic Chemistry-Breakthroughs and Perspectives, Eds.: Ding, K.; Dai, L.-X., Wiley-VCH, Weinheim, 2012.

(g) Liang, T.; Neumann, C. N.; Ritter, T. Angew. Chem., Int. Ed. 2013, 52, 8214.

(h) Chu, L.; Qing, F.-L. Acc. Chem. Res. 2014, 47, 1513.

(i) Alonso, C.; Martínez de Marigorta, E.; Rubiales, G.; Palacios, F. Chem. Rev. 2015, 115, 1847.

(j) Charpentier, J.; Früh, N.; Togni, A. Chem. Rev. 2015, 115, 650.

(k) Huang, L.; Zheng, S.-C.; Tan, B.; Liu, X.-Y. Chem.-Eur. J. 2015, 21, 6718 .

(1) Xu, X.-H.; Matsuzaki, K.; Shibata, N. Chem. Rev. 2015, 115, 731 .

(m) Yang, X.; Wu, T.; Phipps, R. J.; Toste, F. D. Chem. Rev. 2015, 115,826 .

(n) Groult, H.; Leroux, F. R.; Tressaud, A. Modern Synthesis Processes and Reactivity of Fluorinated Compounds, Elsevier Inc., 2017.

(o) Hui, R.; Zhang, S.; Tan, Z.; Wu, X.; Feng, B. Chin. J. Org. Chem. 2017, 37, 3060 (in Chinese).

(惠人杰，张士伟，谭政，吴小培，冯柏年，有机化学，2011，37, 3060.)

(p) Song, H.-X.; Han, Q.-Y.; Zhao, C.-L.; Zhang, C.-P. Green Chem. 2018, 20, 1662.

[7] (a) Magueur, G.; Crousse, B.; Charneau, S.; Grellier, P.; Bégué, J.-P.; Bonnet-Delpon, D. J. Med. Chem. 2004, 47, 2694.

(b) Frezza, M.; Balestrino, D.; Soulère, L.; Reverchon, S.; Queneau, Y.; Forestier, C.; Doutheau, A. Eur. J. Org. Chem. 2006, 2006, 4731.

(c) Chen, J.-L.; You, Z.-W.; Qing, F.-L. J. Fluorine Chem. 2013, $155,143$.

(d) Kim, S.; Kim, E.; Lee, W.; Hong, J. H. Nucleosides Nucleotides Nucleic Acids 2014, 33, 747.

(e) Kollatos, N.; Manta, S.; Dimopoulou, A.; Parmenopoulou, V.; Triantakonstanti, V. V.; Kellici, T.; Mavromoustakos, T.; Schols, D.; Komiotis, D. Carbohydr. Res. 2015, 407, 170.

(f) Shibata, H.; Tsuchikawa, H.; Hayashi, T.; Matsumori, N.; Murata, M.; Usui, T. Chem.-Asian J. 2015, 10, 915.

(g) Achmatowicz, M. M.; Allen, J. G.; Bio, M. M.; Bartberger, M. D.; Borths, C. J.; Colyer, J. T.; Crockett, R. D.; Hwang, T.-L.; Koek, J. N.; Osgood, S. A.; Roberts, S. W.; Swietlow, A.; Thiel, O. R.; Caille, S. J. Org. Chem. 2016, 81, 4736.

[8] (a) Yang, B.; Xu, X.-H.; Qing, F.-L. Chin. J. Chem. 2016, 34, 465. (b) Li, T.; Yu, P.; Lin, J.-S.; Zhi, Y.; Liu, X.-Y. Chin. J. Chem. 2016, $34,490$.

[9] (a) Zhu, R.; Buchwald, S. L. J. Am. Chem. Soc. 2012, 134, 12462. (b) Beniazza, R.; Molton, F.; Duboc, C.; Tron, A.; McClenaghan, N. D.; Lastécouères, D.; Vincent, J.-M. Chem. Commun. 2015, 51, 9571.

(c) Wang, Y.; Jiang, M.; Liu, J.-T. Adv. Synth. Catal. 2016, 358, 1322.

(d) Cheng, Y.-F.; Dong, X.-Y.; Gu, Q.-S.; Yu, Z.-L.; Liu, X.-Y. Angew. Chem., Int. Ed. 2017, 56, 8883.

[10] Noto, N.; Koike, T.; Akita, M. J. Org. Chem. 2016, 81, 7064.

[11] (a) Foulard, G.; Brigaud, T.; Portella, C. J. Fluorine Chem. 1998, 91, 179.

(b) Lin, R.; Sun, H.; Yang, C.; Shen, W.; Xia, W. Chem. Commun. 2015, 51, 399.

(c) Ryzhakov, D.; Jarret, M.; Guillot, R.; Kouklovsky, C.; Vincent, G. Org. Lett. 2017, 19, 6336. 
[12] (a) Heaton, C. A.; Powell, R. L. J. Fluorine Chem. 1989, 45, 86. (b) Kamigata, N.; Fukushima, T.; Yoshida, M. J. Chem. Soc., Chem. Commun. 1989, 1559.

(c) Nagib, D. A.; MacMillan, D. W. C. Nature 2011, 480, 224.

(d) Ni, C.; Hu, M.; Hu, J. Chem. Rev. 2015, 115, 765.

(e) Chachignon, H.; Guyon, H.; Cahard, D. Beilstein J. Org. Chem. 2017, 13, 2800.

[13] (a) Yu, P.; Lin, J.-S.; Li, L.; Zheng, S.-C.; Xiong, Y.-P.; Zhao, L.-J.; Tan, B.; Liu, X.-Y. Angew. Chem., Int. Ed. 2014, 53, 11890.

(b) Yu, P.; Zheng, S.-C.; Yang, N.-Y.; Tan, B.; Liu, X.-Y. Angew. Chem., Int. Ed. 2015, 54, 4041.

(c) Lin, J.-S.; Dong, X.-Y.; Li, T.-T.; Jiang, N.-C.; Tan, B.; Liu, X.-Y. J. Am. Chem. Soc. 2016, 138, 9357.

(d) Li, L.; Li, Z.-L.; Wang, F.-L.; Guo, Z.; Cheng, Y.-F.; Wang, N.; Dong, X.-W.; Fang, C.; Liu, J.; Hou, C.; Tan, B.; Liu, X.-Y. Nat. Commun. 2016, 7, 13852.

(e) Li, L.; Li, Z.-L.; Gu, Q.-S.; Wang, N.; Liu, X.-Y. Sci. Adv. 2017, 3, e1701487.

(f) Lin, J.-S.; Wang, F.-L.; Dong, X.-Y.; He, W.-W.; Yuan, Y.; Chen, S.; Liu, X.-Y. Nat. Commun. 2017, 8, 14841.

(g) Li, X.-T.; Gu, Q.-S.; Dong, X.-Y.; Meng, X.; Liu, X.-Y. Angew. Chem., Int. Ed. 2018, 57, 7668.

[14] (a) Yoon, T. P.; Ischay, M. A.; Du, J. Nat. Chem. 2010, 2, 527.

(b) Narayanam, J. M. R.; Stephenson, C. R. J. Chem. Soc. Rev. 2011, 40, 102 .

(c) Teplý, F. Collect. Czech. Chem. Commun. 2011, 76, 859.

(d) Xuan, J.; Xiao, W.-J. Angew. Chem., Int. Ed. 2012, 51, 6828.

(e) Prier, C. K.; Rankic, D. A.; MacMillan, D. W. C. Chem. Rev. 2013, 113, 5322 .

(f) Hopkinson, M. N.; Sahoo, B.; Li, J.-L.; Glorius, F. Chem.-Eur. J.
2014, 20, 3874.

(g) Koike, T.; Akita, M. Inorg. Chem. Front. 2014, 1, 562

(h) Nicewicz, D. A.; Nguyen, T. M. ACS Catal. 2014, 4, 355.

(i) Ravelli, D.; Protti, S.; Fagnoni, M. Chem. Rev. 2016, 116, 9850.

(j) Romero, N. A.; Nicewicz, D. A. Chem. Rev. 2016, 116, 10075.

(k) Shaw, M. H.; Twilton, J.; MacMillan, D. W. C. J. Org. Chem. 2016, 81,6898 .

(1) Skubi, K. L.; Blum, T. R.; Yoon, T. P. Chem. Rev. 2016, 116, 10035 .

(m) Staveness, D.; Bosque, I.; Stephenson, C. R. J. Acc. Chem. Res. 2016, 49, 2295.

(n) Twilton, J.; Le, C.; Zhang, P.; Shaw, M. H.; Evans, R. W.; MacMillan, D. W. C. Nat. Rev. Chem. 2017, 1, 52.

(o) Cao, M.-Y.; Ren, X.; Lu, Z. Tetrahedron Lett. 2015, 56, 3732.

[15] (a) Huang, L.; Ye, L.; Li, X.-H.; Li, Z.-L.; Lin, J.-S.; Liu, X.-Y. Org. Lett. 2016, 18, 5284.

(b) Li, Z.-L.; Li, X.-H.; Wang, N.; Yang, N.-Y.; Liu, X.-Y. Angew. Chem., Int. Ed. 2016, 55, 15100 .

(c) Wang, N.; Li, L.; Li, Z.-L.; Yang, N.-Y.; Guo, Z.; Zhang, H.-X.; Liu, X.-Y. Org. Lett. 2016, 18, 6026.

(d) Wang, N.; Wang, J.; Guo, Y.-L.; Li, L.; Sun, Y.; Li, Z.; Zhang, H.-X.; Guo, Z.; Li, Z.-L.; Liu, X.-Y. Chem. Commun. 2018, 54, 8885 .

[16] Kim, E.; Choi, S.; Kim, H.; Cho, E. J. Chem.-Eur. J. 2013, 19, 6209.

[17] (a) Jiang, H.; Huang, C.; Guo, J.; Zeng, C.; Zhang, Y.; Yu, S. Chem.-Eur. J. 2012, 18, 15158.

(b) Jiang, H.; Cheng, Y.; Zhang, Y.; Yu, S. Eur. J. Org. Chem. 2013 2013,5485 . 